\title{
4-Dimensional Euler-Totient Matrix Operator and Some Double Sequence Spaces
}

\author{
Sezer Erdem and Serkan Demiriz*
}

\begin{abstract}
Our main purpose in this study is to investigate the matrix domains of the 4-dimensional Euler-totient matrix operator on the classical double sequence spaces $\mathcal{M}_{u}, \mathcal{C}_{p}, \mathcal{C}_{b p}$ and $\mathcal{C}_{r}$. Besides these, we examine their topological and algebraic properties and give inclusion relations about the new spaces. Also, the $\alpha-$, $\beta(\vartheta)-$ and $\gamma$-duals of these spaces are determined and finally, some matrix classes are characterized.

Keywords: Euler function, Möbius function, 4-dimensional Euler-totient matrix operator, matrix domain, double sequence space, $\alpha-, \beta(\vartheta)$ - and $\gamma$-duals, matrix transformations.

AMS Subject Classification (2020): Primary: 40C05; Secondary: 46A45; 47B37.

${ }^{*}$ Corresponding author
\end{abstract}

\section{Preliminaries, Background and Notations}

The function $f$ described by $f: \mathbb{N} \times \mathbb{N} \rightarrow \wp,(t, u) \mapsto f(t, u)=x_{t u}$ is entitled as double sequence, where $\wp$ denotes any nonempty set and $\mathbb{N}=\{1,2, \ldots\} . \Omega$ stands for the set of all complex valued double sequences. It is well known that this set is a vector space with coordinatewise addition and scalar multiplication. Any linear subspace of $\Omega$ is called as double sequence space. The set of all bounded complex valued double sequences is symbolized with $\mathcal{M}_{u}$, that is,

$$
\mathcal{M}_{u}=\left\{x=\left(x_{t u}\right) \in \Omega:\|x\|_{\infty}=\sup _{t, u \in \mathbb{N}}\left|x_{t u}\right|<\infty\right\} .
$$

It should be noted that $\mathcal{M}_{u}$ is a Banach space with the norm $\|x\|_{\infty}$. We say that the double sequence $x=\left(x_{t u}\right)$ is convergent in the Pringsheim's sense provided that for every $\varepsilon>0$ there exists $n_{\varepsilon} \in \mathbb{N}$ such that $\left|x_{t u}-L\right|<\varepsilon$ whenever $t, u>n_{\varepsilon}$. In that case, $L \in \mathbb{C}$ is called the Pringsheim limit of $x$ and stated by $p-\lim _{t, u \rightarrow \infty} x_{t u}=L$; where $\mathbb{C}$ denotes the complex field. $\mathcal{C}_{p}$ represents the space of all such $x$ which are called shortly as $p$-convergent. Of particular interest is unlike single sequences, $p$-convergent double sequences need not be bounded. For example, if we consider the sequence $x=\left(x_{t u}\right)$ identified by

$$
x_{t u}=\left(\begin{array}{cccccc}
1 & 2 & 3 & \cdots & u & \cdots \\
2 & 0 & 0 & \cdots & 0 & \cdots \\
3 & 0 & 0 & \cdots & 0 & \cdots \\
\vdots & \vdots & \vdots & \ldots & \vdots & \ldots \\
t & 0 & 0 & \cdots & 0 & \cdots \\
\vdots & \vdots & \vdots & \ldots & \vdots & \ldots
\end{array}\right),
$$

it can easily seen that $p-\lim x_{t u}=0$ but $\|x\|_{\infty}=\infty$. As a conclusion $x \in \mathcal{C}_{p}-\mathcal{M}_{u}$. The bounded sequences which are also $p$-convergent are indicated by $\mathcal{C}_{b p}$, that is, $\mathcal{C}_{b p}=\mathcal{C}_{p} \cap \mathcal{M}_{u}$. A double sequence $x=\left(x_{t u}\right) \in \mathcal{C}_{p}$ is called as regularly convergent if the limits $x_{t}:=\lim _{u} x_{t u},(t \in \mathbb{N})$ and $x_{u}:=\lim _{t} x_{t u},(u \in \mathbb{N})$ exist, and the $\operatorname{limits}_{\lim _{t}} \lim _{u} x_{t u}$

Received : 06-05-2020, Accepted : 12-07-2020 
and $\lim _{u} \lim _{t} x_{t u}$ exist and are equivalent to the $p-\lim$ of $x$. The space of all regularly convergent double sequences is denoted by $\mathcal{C}_{r}$. Obviously, the regular convergence of a double sequence $x$ implies the convergence in Pringsheim's sense as well as the boundedness of the terms of $x$, but the converse implication fails. A sequence $x=\left(x_{t u}\right)$ is called double null sequence if it converges to zero. Additionally, all double null sequences in the spaces $\mathcal{C}_{b p}$ and $\mathcal{C}_{r}$ are denoted by $\mathcal{C}_{b p 0}$ and $\mathcal{C}_{r 0}$, respectively. Móricz [25] showed that the spaces $\mathcal{C}_{b p}, \mathcal{C}_{b p 0}, \mathcal{C}_{r}$ and $\mathcal{C}_{r 0}$ are Banach spaces endowed with the norm $\|\cdot\|_{\infty}$.

Let us take any $x \in \Omega$ and describe the sequence $K=\left(k_{r s}\right)$ defined by

$$
k_{r s}:=\sum_{t=1}^{r} \sum_{u=1}^{s} x_{t u}, \quad(r, s \in \mathbb{N}) .
$$

In that case, the pair $\left(\left(x_{r s}\right),\left(k_{r s}\right)\right)$ is entitled as double series. Here, the sequence $K=\left(k_{r s}\right)$ is the sequence of partial sums of the double series.

Consider the double sequence space $\Psi$ converging with respect to some linear convergence rule $\vartheta-\lim : \Psi \rightarrow \mathbb{C}$. The sum of a double series $\sum_{t, u} x_{t u}$ relating to this rule is defined by $\vartheta-\sum_{t, u} x_{t u}=\vartheta-\lim _{r, s \rightarrow \infty} s_{r s}$. Here and thereafter, when needed we will use the summation $\sum_{t, u}$ instead of $\sum_{t=1}^{\infty} \sum_{u=1}^{\infty}$, assume that $\vartheta \in\{p, b p, r\}$ and $p^{\prime}$ denotes the conjugate of $p$, that is, $p^{\prime}=p /(p-1)$ for $1<p<\infty$. With the notation of Zeltser [42], we describe the double sequences $e^{r s}=\left(e_{t u}^{r s}\right)$ and $e$ by $e_{t u}^{r s}=1$ if $(r, s)=(t, u)$ and $e_{t, u}^{r, s}=0$ otherwise, and $e=\sum_{r, s} e^{r, s}$ (coordinatewise convergence) for every $r, s, t, u \in \mathbb{N}$.

The $\alpha$-dual $\Psi^{\alpha}, \beta(\vartheta)$-dual $\Psi^{\beta(\vartheta)}$ with respect to the $\vartheta$-convergence and the $\gamma$-dual $\Psi^{\gamma}$ of a double sequence space $\Psi$ are described as

$$
\begin{aligned}
\Psi^{\alpha} & :=\left\{c=\left(c_{t u}\right) \in \Omega: \sum_{t, u}\left|c_{t u} x_{t u}\right|<\infty \text { for all }\left(x_{t u}\right) \in \Psi\right\}, \\
\Psi^{\beta(\vartheta)} & :=\left\{c=\left(c_{t u}\right) \in \Omega: \vartheta-\sum_{t, u} c_{t u} x_{t u} \text { exists for all }\left(x_{t u}\right) \in \Psi\right\}, \\
\Psi^{\gamma} & :=\left\{c=\left(c_{t u}\right) \in \Omega: \sup _{r, s \in \mathbb{N}}\left|\sum_{t, u=1}^{r, s} c_{t u} x_{t u}\right|<\infty \text { for all }\left(x_{t u}\right) \in \Psi\right\},
\end{aligned}
$$

respectively. It is well known that $\Psi^{\alpha} \subset \Psi^{\gamma}$ and if $\Psi \subset \Lambda$, then $\Lambda^{\alpha} \subset \Psi^{\alpha}$ for the double sequence spaces $\Psi$ and $\Lambda$.

Let us remember the definition of triangle matrix. If $b_{r s t u}=0$ for $t>r$ or $u>s$ or both for every $r, s, t, u \in \mathbb{N}$, it is said that $B=\left(b_{r s t u}\right)$ is a triangular matrix and also if $b_{r s r s} \neq 0$ for every $r, s \in \mathbb{N}$, then the 4-dimensional matrix $B$ is called triangle. It should be noted by [11] that, every triangle has a unique inverse which is also a triangle.

Now, we shall deal with matrix mapping. Let us consider double sequence spaces $\Psi$ and $\Lambda$ and the 4-dimensional complex infinite matrix $B=\left(b_{r s t u}\right)$. In that case, we say that $B$ defines a matrix mapping from $\Psi$ into $\Lambda$ and it is written as $B: \Psi \rightarrow \Lambda$, if for every sequence $x=\left(x_{t u}\right) \in \Psi$, the $B$-transform $B x=\left\{(B x)_{r s}\right\}_{r, s \in \mathbb{N}}$ of $x$ exists and is in $\Lambda$; where

$$
(B x)_{r s}=\vartheta-\sum_{t, u} b_{r s t u} x_{t u},
$$

for each $r, s \in \mathbb{N}$. ( $\Psi: \Lambda)$ stands for the class of all 4-dimensional complex infinite matrices from a double sequence space $\Psi$ into a double sequence space $\Lambda$. In that case, $B \in(\Psi: \Lambda)$ if and only if $B_{r s} \in \Psi^{\beta(\vartheta)}$, where $B_{r s}=\left(b_{r s t u}\right)_{t, u \in \mathbb{N}}$ for all $r, s \in \mathbb{N}$.

The $\vartheta$-summability domain $\Psi_{B}^{(\vartheta)}$ of a 4-dimensional complex infinite matrix $B$ in a double sequence space $\Psi$ consists of whose $B$-transforms are in $\Psi$; that is,

$$
\Psi_{B}^{(\vartheta)}:=\left\{x=\left(x_{t u}\right) \in \Omega: B x:=\left(\vartheta-\sum_{t u} b_{r s t u} x_{t u}\right)_{r, s \in \mathbb{N}} \text { exists and is in } \Psi\right\} .
$$

In the past, many authors were interested in double sequence spaces. Now, let us give some information about these studies. Zeltser [41] has fundamentally examined both the topological structure and the theory of summability of double sequences in her doctoral dissertation. Recently, Altay and Başar [3] defined the double sequence spaces $\mathcal{B S}, \mathcal{B S}(t), \mathcal{C S}_{p}, \mathcal{C S}_{b p}, \mathcal{C} \mathcal{S}_{r}$ and $\mathcal{B V}$ of double series whose sequences of partial sums are in the spaces $\mathcal{M}_{u}, \mathcal{M}_{u}(t)$, $\mathcal{C}_{p}, \mathcal{C}_{b p}, \mathcal{C}_{r}$ and $\mathcal{L}_{u}$, respectively, and also examined some properties of those spaces. Later, in [5], Başar and Sever 
have defined the set $\mathcal{L}_{p}$ of all absolutely $p$-summable double sequences which is a Banach space with the norm $\|\cdot\|_{\mathcal{L}_{p}}$ defined in the following way:

$$
\|\cdot\|_{\mathcal{L}_{p}}=\left(\sum_{t, u}\left|x_{t u}\right|^{p}\right)^{\frac{1}{p}} .
$$

It is also significant that the double sequence space $\mathcal{L}_{u}$ which was defined by Zeltser [42] is the special case of the space $\mathcal{L}_{p}$ for $p=1$. For more details about the double sequences and related topics, the reader may refer to $[1,3-5,13,25-28,31,35-40,43]$ and references therein.

In the rest of the study, $\varphi$ and $\mu$ represent Euler function and the Möbius function, respectively. For every $r \in \mathbb{N}$ with $r>1, \varphi(r)$ is the number of positive integers less than $r$ which are coprime with $r$ and $\varphi(1)=1$. If $a_{1}{ }^{b_{1}} a_{2}{ }^{b_{2}} a_{3}{ }^{b_{3}} \ldots a_{m}{ }^{b_{m}}$ is the prime factorization of a natural number $r>1$, then

$$
\varphi(r)=r\left(1-\frac{1}{a_{1}}\right)\left(1-\frac{1}{a_{2}}\right)\left(1-\frac{1}{a_{3}}\right) \ldots\left(1-\frac{1}{a_{m}}\right) .
$$

Also, the equality

$$
r=\sum_{t \mid r} \varphi(t)
$$

holds for every $r \in \mathbb{N}$ and $\varphi\left(r_{1} r_{2}\right)=\varphi\left(r_{1}\right) \varphi\left(r_{2}\right)$, where $r_{1}, r_{2} \in \mathbb{N}$ are coprime. Given any $r \in \mathbb{N}$ with $r>1, \mu$ is defined as

$$
\mu(r):=\left\{\begin{array}{cc}
(-1)^{m}, & \text { if } r=a_{1} a_{2} \ldots a_{m}, \text { where } a_{1} a_{2} \ldots a_{m} \text { are } \\
& \text { non-equivalent prime numbers } \\
0 \quad, \quad & \text { if } a^{2} \mid r \text { for some prime number } \quad a,
\end{array}\right.
$$

and $\mu(1)=1$. If $a_{1}{ }^{b_{1}} a_{2}{ }^{b_{2}} a_{3}{ }^{b_{3}} \ldots a_{m}{ }^{b_{m}}$ is the prime factorization of a naturel number $r>1$, in this fact,

$$
\sum_{t \mid r} t \mu(t)=\left(1-a_{1}\right)\left(1-a_{2}\right)\left(1-a_{3}\right) \ldots\left(1-a_{m}\right) .
$$

If $r \neq 1$, then the equality

$$
\sum_{t \mid r} \mu(t)=0
$$

holds and $\mu\left(r_{1} r_{2}\right)=\mu\left(r_{1}\right) \mu\left(r_{2}\right)$, where $r_{1}, r_{2} \in \mathbb{N}$ are coprime.

By using the regular 2-dimensional Euler-totient matrix $\Phi$, the Euler-totient sequence spaces $\ell_{p}(\Phi)$ and $\ell_{\infty}(\Phi)$ which consist of all sequences whose $\Phi$-transforms are in the spaces $\ell_{p}$ of absolutely $p$-summable and $\ell_{\infty}$ of bounded single sequences are introduced and examined by Illkhan and Kara [18].

The target of the existing study is to acquaint the matrix domains of the 4-dimensional Euler-totient matrix on some classical double sequence spaces.

\section{Domain of Euler-Totient Matrix in Some Spaces of Double Sequences}

In this section, we introduce the double sequence spaces $\Phi^{\star}\left(\mathcal{M}_{u}\right), \Phi^{\star}\left(\mathcal{C}_{p}\right), \Phi^{\star}\left(\mathcal{C}_{b p}\right)$ and $\Phi^{\star}\left(\mathcal{C}_{r}\right)$ by using the 4-dimensional Euler-totient matrix $\Phi^{\star}$ and give some properties and results on theese spaces.

In [14], we have defined the 4-dimensional matrix $\Phi^{\star}=\left(\phi_{r s t u}^{\star}\right)$ which is called Euler-totient matrix operator as follows:

$$
\phi_{\text {rstu }}^{\star}:=\left\{\begin{array}{cc}
\frac{\varphi(t) \varphi(u)}{r s}, & t|r, u| s, \\
0 \quad, & \text { otherwise, }
\end{array}\right.
$$

for every $r, s, t, u \in \mathbb{N}$. Thus, it is clear that $\Phi^{\star}$ is a triangle and the $\Phi^{\star}$-transform of a double sequence $x=\left(x_{r s}\right)$ is given by

$$
y_{r s}:=\left(\Phi^{\star} x\right)_{r s}=\frac{1}{r s} \sum_{t|r, u| s} \varphi(t) \varphi(u) x_{t u}
$$


for every $r, s \in \mathbb{N}$. Throughout the article, we suppose that the terms of the double sequences $x=\left(x_{r s}\right)$ and $y=\left(y_{r s}\right)$ are connected with the relation (2.2).

The inverse $\left(\Phi^{\star}\right)^{-1}=\left(\phi_{r s t u}^{\star-1}\right)$ of the triangle matrix $\Phi^{\star}$ is calculated as

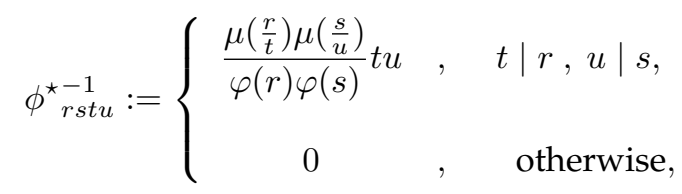

for every $r, s, t, u \in \mathbb{N}$. We introduce the sequence spaces $\Phi^{\star}\left(\mathcal{M}_{u}\right), \Phi^{\star}\left(\mathcal{C}_{p}\right), \Phi^{\star}\left(\mathcal{C}_{b p}\right)$ and $\Phi^{\star}\left(\mathcal{C}_{r}\right)$ as the sets of all double sequences such that $\Phi^{\star}$-transforms of them are in the spaces $\mathcal{M}_{u}, \mathcal{C}_{p}, \mathcal{C}_{b p}$ and $\mathcal{C}_{r}$, that is,

$$
\begin{aligned}
\Phi^{\star}\left(\mathcal{M}_{u}\right) & =\left\{x=\left(x_{r s}\right) \in \Omega: \sup _{r, s \in \mathbb{N}}\left|\frac{1}{r s} \sum_{t|r, u| s} \varphi(t) \varphi(u) x_{t u}\right|<\infty\right\}, \\
\Phi^{\star}\left(\mathcal{C}_{p}\right) & =\left\{x=\left(x_{r s}\right) \in \Omega: \exists L \in \mathbb{C} \ni p-\lim _{r, s \rightarrow \infty}\left|\frac{1}{r s} \sum_{t|r, u| s} \varphi(t) \varphi(u) x_{t u}-L\right|=0\right\}, \\
\Phi^{\star}\left(\mathcal{C}_{b p}\right) & =\left\{x=\left(x_{r s}\right) \in \Omega:\left(\frac{1}{r s} \sum_{t|r, u| s} \varphi(t) \varphi(u) x_{t u}\right) \in \mathcal{C}_{b p}\right\}, \\
\Phi^{\star}\left(\mathcal{C}_{r}\right) & =\left\{x=\left(x_{r s}\right) \in \Omega:\left(\frac{1}{r s} \sum_{t|r, u| s} \varphi(t) \varphi(u) x_{t u}\right) \in \mathcal{C}_{r}\right\} .
\end{aligned}
$$

It is immediately seen that $\Phi^{\star}\left(\mathcal{M}_{u}\right), \Phi^{\star}\left(\mathcal{C}_{p}\right), \Phi^{\star}\left(\mathcal{C}_{b p}\right)$ and $\Phi^{\star}\left(\mathcal{C}_{r}\right)$ are the domains of the 4-dimensional Eulertotient matrix $\Phi^{\star}$ in the spaces $\mathcal{M}_{u}, \mathcal{C}_{p}, \mathcal{C}_{b p}$ and $\mathcal{C}_{r}$, respectively.

If $\Psi$ is any normed double sequence space, then we call the matrix domain $\Phi^{\star}(\Psi)$ as the double Euler-totient sequence space.

Definition 2.1 (See [16],[31]). A 4-dimensional matrix $B$ is said to be RH-regular if it maps every bounded $p$ convergent sequence into a $p$-convergent sequence with the same $p$-limit.

Lemma 2.1 (See [16],[31]). A 4-dimensional triangle matrix $B=\left(b_{r s t u}\right)$ is RH-regular iff

$$
\begin{aligned}
& R H_{1}: p-\lim _{r, s \rightarrow \infty} b_{r s t u}=0 \quad \text { for each } t, u \in \mathbb{N}, \\
& R H_{2}: p-\lim _{r, s \rightarrow \infty} \sum_{t, u} b_{r s t u}=1, \\
& R H_{3}: p-\lim _{r, s \rightarrow \infty} \sum_{t}\left|b_{r s t u}\right|=0 \text { for each } u \in \mathbb{N}, \\
& R H_{4}: p-\lim _{r, s \rightarrow \infty} \sum_{u}\left|b_{r s t u}\right|=0 \text { for each } t \in \mathbb{N}, \\
& R H_{5}: \text { There exists finite positive integers } M_{1} \text { and } M_{2} \text { such that } \\
& \sum_{t, u>M_{1}}\left|b_{r s t u}\right|<M_{2} .
\end{aligned}
$$

It should be noted that the 4-dimensional Euler-totient matrix $\Phi^{\star}$ described by (2.1) is RH-regular [14].

Now, we may continue with the following two theorems which are the essential in the study.

Theorem 2.1. The sets $\Phi^{\star}\left(\mathcal{M}_{u}\right), \Phi^{\star}\left(\mathcal{C}_{b p}\right)$ and $\Phi^{\star}\left(\mathcal{C}_{r}\right)$ are the linear spaces which are linearly norm isomorphic to the spaces $\mathcal{M}_{u}, \mathcal{C}_{b p}$ and $\mathcal{C}_{r}$, respectively, and are the Banach spaces with the norm

$$
\|x\|_{\Phi^{\star}\left(\mathcal{M}_{u}\right)}=\left\|\Phi^{\star} x\right\|_{\infty}=\sup _{r, s \in \mathbb{N}}\left|\frac{1}{r s} \sum_{t|r, u| s} \varphi(t) \varphi(u) x_{t u}\right| .
$$


Proof. To avoid the repetition of the similar statements, we give the proof only for the space $\Phi^{\star}\left(\mathcal{M}_{u}\right)$. Since the initial assertion is routine verification and is easy to prove, we ignore its proof in here. To confirm the fact that $\Phi^{\star}\left(\mathcal{M}_{u}\right)$ is linearly norm isomorphic to the space $\mathcal{M}_{u}$, we need to be sure the existence of a linear and norm preserving bijection between the spaces $\Phi^{\star}\left(\mathcal{M}_{u}\right)$ and $\mathcal{M}_{u}$. For this purpose, let us take the transformation $B$ defined from $\Phi^{\star}\left(\mathcal{M}_{u}\right)$ into $\mathcal{M}_{u}$ by $x \mapsto y=B x$, where $y=\left(y_{r s}\right)$ is the $\Phi^{\star}$-transform of the sequence $x=\left(x_{t u}\right)$. The linearity of $B$ is clear. Consider the equality $B x=\theta$ which yields us that $x_{t u}=0$ for every $t, u \in \mathbb{N}$. So, $x=\theta$. Therefore, $B$ is injective. Let us consider $y \in \mathcal{M}_{u}$ and describe the double sequence $x=\left(x_{r s}\right)$ by

$$
x_{r s}=\sum_{t|r, u| s} \frac{\mu\left(\frac{r}{t}\right) \mu\left(\frac{s}{u}\right)}{\varphi(r) \varphi(s)} t_{u y_{t u}}
$$

for every $r, s \in \mathbb{N}$. By taking supremum over $r, s \in \mathbb{N}$ on the following equality

$$
\left|\left(\Phi^{\star} x\right)_{r s}\right|=\left|\frac{1}{r s} \sum_{t|r, s| u} \varphi(t) \varphi(u) x_{t u}\right|=\left|y_{r s}\right|,
$$

it can be derived that $B$ is surjective and norm preserving.

Now, we may prove that $\Phi^{\star}\left(\mathcal{M}_{u}\right)$ is a Banach space with the norm $\|\cdot\|_{\Phi^{\star}\left(\mathcal{M}_{u}\right)}$ described by (2.3). Since $\mathcal{M}_{u}$ is a Banach space from [25], we obtain the desired result from Section (b) of Corollary 6.3.41 in [6].

Theorem 2.2. The set $\Phi^{\star}\left(\mathcal{C}_{p}\right)$ is linearly isomorphic to the space $\mathcal{C}_{p}$ and is a complete semi-normed space with the semi-norm

$$
\|x\|_{\Phi^{\star}\left(\mathcal{C}_{p}\right)}=\lim _{i \rightarrow \infty}\left(\sup _{r, s \geq i}\left|\left(\Phi^{\star} x\right)_{r s}\right|\right) .
$$

Proof. Since the proof of the theorem is similar to the proof of Theorem 2.1, we ignore it.

Now, let us give our results about inclusion relations.

Theorem 2.3. The inclusion $\mathcal{M}_{u} \subset \Phi^{\star}\left(\mathcal{M}_{u}\right)$ holds.

Proof. Let us take a sequence $x=\left(x_{t u}\right) \in \mathcal{M}_{u}$. In that case, there exists a positive real number $M_{3}$ such that $\sup _{t, u \in \mathbb{N}}\left|x_{t u}\right| \leq M_{3}$. Therefore, one can immediately see that

$$
\begin{aligned}
\|x\|_{\Phi^{\star}\left(\mathcal{M}_{u}\right)} & =\sup _{r, s \in \mathbb{N}}\left|\frac{1}{r s} \sum_{t|r, u| s} \varphi(t) \varphi(u) x_{t u}\right| \\
& \leq \sup _{r, s \in \mathbb{N}}\left|\frac{1}{r s} \sum_{t|r, u| s} \varphi(t) \varphi(u)\right|\left|x_{t u}\right| \\
& \leq M_{3} \sup _{r, s \in \mathbb{N}}\left|\frac{1}{r s} \sum_{t|r, u| s} \varphi(t) \varphi(u)\right|=M_{3} .
\end{aligned}
$$

Thus, the inclusion is valid.

Theorem 2.4. The inclusion $\mathcal{C}_{b p} \subset \Phi^{\star}\left(\mathcal{C}_{p}\right)$ holds.

Proof. Let us take the sequence $x=\left(x_{t u}\right) \in \mathcal{C}_{b p}$ with $p \lim _{t, u \rightarrow \infty} x_{t u}=L$. Since 4-dimensional Euler-totient matrix is RH-regular, $p \lim _{t, u \rightarrow \infty} y_{t u}=L$, where $\left(y_{t u}\right)=\left(\Phi^{\star} x\right)_{t u}$. Hence, we see that $\mathcal{C}_{b p} \subset \Phi^{\star}\left(\mathcal{C}_{p}\right)$. 


\section{Dual Spaces}

In the current section, we tend to compute the $\alpha-, \beta(\vartheta)-$ and $\gamma$-duals of the new double Euler-totient sequence spaces.

Theorem 3.1. The $\alpha$-dual of the space $\Phi^{\star}\left(\mathcal{M}_{u}\right)$ is $\mathcal{L}_{u}$.

Proof. Suppose that $c=\left(c_{r s}\right) \in\left\{\Phi^{\star}\left(\mathcal{M}_{u}\right)\right\}^{\alpha}$ but $c \notin \mathcal{L}_{u}$. Then, $\sum_{r, s}\left|c_{r s} x_{r s}\right|<\infty$ for all $x=\left(x_{r s}\right) \in \Phi^{\star}\left(\mathcal{M}_{u}\right)$. If we consider $e \in \Phi^{\star}\left(\mathcal{M}_{u}\right)$, in that case $c e=c \notin \mathcal{L}_{u}$, that is $c \notin\left\{\Phi^{\star}\left(\mathcal{M}_{u}\right)\right\}^{\alpha}$ and it is seen that this is a contradiction. Thus, $c$ must be in $\mathcal{L}_{u}$.

Conversely, let us take sequences $c=\left(c_{r s}\right) \in \mathcal{L}_{u}$ and $x=\left(x_{r s}\right) \in \Phi^{\star}\left(\mathcal{M}_{u}\right)$. In that case, there exists a double sequence $y=\left(y_{r s}\right) \in \mathcal{M}_{u}$ such that $y=\Phi^{\star} x$ and $\sup _{r, s}\left|y_{r s}\right|<M_{4}$, where $M_{4} \in \mathbb{R}^{+}$. Then, we have from the following inequality

$$
\begin{aligned}
\sum_{r, s}\left|c_{r s} x_{r s}\right| & =\sum_{r, s}\left|c_{r s}\right|\left|\sum_{t|r, u| s} \frac{\mu\left(\frac{r}{t}\right) \mu\left(\frac{s}{u}\right)}{\varphi(r) \varphi(s)} t u y_{t u}\right| \\
& \leq M_{4} \sum_{r, s}\left|c_{r s}\right|\left|\sum_{t|r, u| s} \frac{\mu\left(\frac{r}{t}\right) \mu\left(\frac{s}{u}\right)}{\varphi(r) \varphi(s)} t u\right| \\
& =M_{4} \sum_{r, s}\left|c_{r s}\right|<\infty
\end{aligned}
$$

that $c \in\left(\Phi^{\star}\left(\mathcal{M}_{u}\right)\right)^{\alpha}$ and this completes the proof.

Now, we give some lemmas which characterize the classes of 4-dimensional matrix mappings(see [16], [42] and [43]). With the help of these lemmas, we will calculate the $\beta(\vartheta), \beta(b p), \beta(p)$ and $\gamma$-duals of our new double sequence spaces.

Lemma 3.1. Suppose that $B=\left(b_{r s t u}\right)$ is a 4-dimensional infinite matrix. Then, $B \in\left(\mathcal{C}_{b p}: \mathcal{C}_{\vartheta}\right)$ iff following conditions hold:

$$
\begin{aligned}
& \sup _{r, s \in \mathbb{N}} \sum_{t, u}\left|b_{r s t u}\right|<\infty, \\
& \exists b_{t u} \in \mathbb{C} \ni \vartheta-\lim _{r, s \rightarrow \infty} b_{r s t u}=b_{t u} \text { for all } \quad t, u \in \mathbb{N}, \\
& \exists L \in \mathbb{C} \ni \vartheta-\lim _{r, s \rightarrow \infty} \sum_{t, u} b_{r s t u}=L \quad \text { exists, } \\
& \exists t_{0} \in \mathbb{N} \ni \vartheta-\lim _{r, s \rightarrow \infty} \sum_{u}\left|b_{r s t_{0} u}-b_{t_{0} u}\right|=0, \\
& \exists u_{0} \in \mathbb{N} \ni \vartheta-\lim _{r, s \rightarrow \infty} \sum_{t}\left|b_{r s t u_{0}}-b_{t u_{0}}\right|=0 .
\end{aligned}
$$

In the case of (3.5), $b=\left(b_{t u}\right) \in \mathcal{L}_{u}$ and

$$
\vartheta-\lim _{r, s \rightarrow \infty}[B x]_{r s}=\sum_{t, u} b_{t u} x_{t u}+\left(L-\sum_{t, u} b_{t u}\right) b p-\lim _{r, s \rightarrow \infty} x_{r s}
$$

satisfies for $x \in \mathcal{C}_{b p}$.

Lemma 3.2. Suppose that $B=\left(b_{r s t u}\right)$ is a 4-dimensional infinite matrix. Then, $B \in\left(\mathcal{C}_{p}: \mathcal{C}_{\vartheta}\right)$ iff (3.1)-(3.3) hold and the following conditions hold, too:

$$
\begin{aligned}
& \forall t \in \mathbb{N}, \quad \exists u_{0} \in \mathbb{N} \ni b_{r s t u}=0 \quad \text { for every } \quad u>u_{0} \text { and } r, s \in \mathbb{N}, \\
& \forall u \in \mathbb{N}, \quad \exists t_{0} \in \mathbb{N} \ni b_{r s t u}=0 \quad \text { for every } t>t_{0} \text { and } r, s \in \mathbb{N} .
\end{aligned}
$$

In the case of (3.7), $\exists t_{0}, u_{0} \in \mathbb{N}$ such that $b=\left(b_{t u}\right) \in \mathcal{L}_{u}$ and $\left(b_{t u_{0}}\right)_{t \in \mathbb{N}},\left(b_{t_{0} u}\right)_{u \in \mathbb{N}} \in \zeta$, where $\zeta$ represents the space of every finitely sequences which are non-equivalent zero and

$$
\vartheta-\lim _{r, s \rightarrow \infty}[B x]_{r s}=\sum_{t, u} b_{t u} x_{t u}+\sum_{t}\left(L-\sum_{t, u} b_{t u}\right) p-\lim _{r, s \rightarrow \infty} x_{r s}
$$


satisfies for $x \in \mathcal{C}_{p}$.

Lemma 3.3. Suppose that $B=\left(b_{r s t u}\right)$ is a 4-dimensional infinite matrix. Then, $B \in\left(\mathcal{C}_{r}: \mathcal{C}_{\vartheta}\right)$ iff (3.1)-(3.3) hold and the following conditions hold, too:

$$
\begin{aligned}
& \exists u_{0} \in \mathbb{N} \ni \vartheta-\lim _{r, s \rightarrow \infty} \sum_{t} b_{r s t u_{0}}=\rho_{u_{0}}, \\
& \exists t_{0} \in \mathbb{N} \ni \vartheta-\lim _{r, s \rightarrow \infty} \sum_{u} b_{r s t_{0} u}=\varrho_{t_{0}} .
\end{aligned}
$$

In the case of (3.9), $b=\left(b_{t u}\right) \in \mathcal{L}_{u}$ and $\rho_{u}, \varrho_{t} \in \ell_{1}$ and

$$
\begin{aligned}
\vartheta-\lim _{r, s \rightarrow \infty}[B x]_{r s} & =\sum_{t, u} b_{t u} x_{t u}+\sum_{t}\left(\varrho_{t}-\sum_{u} b_{t u}\right) x_{t}+\sum_{u}\left(\rho_{u}-\sum_{t} b_{t u}\right) x_{u} \\
& +\left(L+\sum_{t, u} b_{t u}-\sum_{t} \varrho_{t}-\sum_{u} \rho_{u}\right) r-\lim _{r, s \rightarrow \infty} x_{r s}
\end{aligned}
$$

satisfies for $x \in \mathcal{C}_{r}$.

Lemma 3.4. [36] Suppose that $B=\left(b_{\text {rstu }}\right)$ is a 4-dimensional infinite matrix. Then, $B \in\left(\mathcal{C}_{b p}: \mathcal{M}_{u}\right)$ iff the condition (3.1) hold.

Lemma 3.5. [12] Suppose that $B=\left(b_{r s t u}\right)$ is a 4-dimensional infinite matrix. Then, $B \in\left(\mathcal{M}_{u}: \mathcal{C}_{b p}\right)$ iff the conditions (3.1), (3.2) hold and the following conditions hold, too:

$$
\begin{aligned}
& \exists b_{t u} \in \mathbb{C} \ni b p-\lim _{r, s \rightarrow \infty} \sum_{t u}\left|b_{r s t u}-b_{t u}\right|=0, \\
& b p-\lim _{r, s \rightarrow \infty} \sum_{u=0}^{s} b_{r s t u} \quad \text { exists for each } \quad t \in \mathbb{N}, \\
& b p-\lim _{r, s \rightarrow \infty} \sum_{t=0}^{r} b_{r s t u} \quad \text { exists for each } \quad u \in \mathbb{N}, \\
& \sum_{t, u}\left|b_{r s t u}\right| \quad \text { converges. }
\end{aligned}
$$

Lemma 3.6. [38] Suppose that $B=\left(b_{r s t u}\right)$ is a 4-dimensional infinite matrix. Then, $B \in\left(\mathcal{M}_{u}: \mathcal{M}_{u}\right)$ iff the condition (3.1) holds.

Lemma 3.7. [39] Suppose that $B=\left(b_{r s t u}\right)$ is a 4-dimensional infinite matrix. Then, $B \in\left(\mathcal{M}_{u}: \mathcal{C}_{p}\right)$ iff the conditions (3.2), (3.6) and (3.7) hold.

Lemma 3.8. [40] Suppose that $B=\left(b_{r s t u}\right)$ is a 4-dimensional infinite matrix. In that case:

(i) If $0<p \leq 1$, then $B \in\left(\mathcal{L}_{p}: \mathcal{M}_{u}\right)$ iff

$$
\sup _{r, s, t, u \in \mathbb{N}}\left|b_{r s t u}\right|<\infty
$$

(ii) If $1<p<\infty$, then $B \in\left(\mathcal{L}_{p}: \mathcal{M}_{u}\right)$ iff

$$
\sup _{r, s \in \mathbb{N}} \sum_{t, u}\left|b_{r s t u}\right|^{p \prime}<\infty .
$$

Lemma 3.9. [40] Suppose that $B=\left(b_{r s t u}\right)$ is a 4-dimensional infinite matrix. In that case:

(i) If $0<p \leq 1$, then $B \in\left(\mathcal{L}_{p}: \mathcal{C}_{b p}\right)$ iff the conditions (3.2) and (3.14) hold with $\vartheta=b p$,

(ii) If $1<p<\infty$, then $B \in\left(\mathcal{L}_{p}: \mathcal{C}_{b p}\right)$ iff the conditions (3.2) and (3.15) hold. 
Theorem 3.2. Consider the set $w_{1}$ defined by

$$
w_{1}=\left\{c=\left(c_{r s}\right) \in \Omega: \sup _{r, s} \sum_{t, u}|\sigma(r, s, t, u, m, n)|<\infty\right\},
$$

where

$$
\sigma(r, s, t, u, m, n)=\sum_{m=t, t \mid m}^{r} \sum_{n=u, u \mid n}^{s} \frac{\mu\left(\frac{m}{t}\right) \mu\left(\frac{n}{u}\right)}{\varphi(m) \varphi(n)} t u c_{m n} .
$$

Then, $\left(\Phi^{\star}\left(\mathcal{C}_{b p}\right)\right)^{\gamma}=w_{1}=\left(\Phi^{\star}\left(\mathcal{M}_{u}\right)\right)^{\gamma}$.

Proof. Suppose that $c=\left(c_{r s}\right) \in \Omega$ and $x=\left(x_{r s}\right) \in \Phi^{\star}\left(\mathcal{C}_{b p}\right)$. Then, we can conclude from (2.2) that $y=\left(y_{r s}\right) \in \mathcal{C}_{b p}$. Now, let us define the 4-dimensional matrix $O=\left(o_{r s t u}\right)$ by

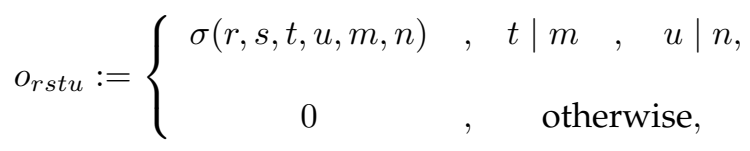

for every $r, s, t, u \in \mathbb{N}$. Therefore, we obtain by using the relation (2.4) that

$$
\begin{aligned}
z_{r s} & =\sum_{t, u=1}^{r, s} c_{t u} x_{t u} \\
& =\sum_{t, u=1}^{r, s} c_{t u}\left[\sum_{m|t, n| u} \frac{\mu\left(\frac{t}{m}\right) \mu\left(\frac{u}{n}\right)}{\varphi(t) \varphi(u)} m n y_{m n}\right] \\
& =\sum_{t, u=1}^{r, s}\left[\sum_{m=t, t \mid m}^{r} \sum_{n=u, u \mid n}^{s} \frac{\mu\left(\frac{m}{t}\right) \mu\left(\frac{n}{u}\right)}{\varphi(m) \varphi(n)} t u c_{m n}\right] y_{t u} \\
& =(O y)_{r s}
\end{aligned}
$$

for every $r, s \in \mathbb{N}$. Then, by considering the equality (3.16), we deduce that $c x=\left(c_{r s} x_{r s}\right) \in \mathcal{B S}$ whenever $x \in \Phi^{\star}\left(\mathcal{C}_{b p}\right)$ iff $z=\left(z_{r s}\right) \in \mathcal{M}_{u}$ whenever $y \in \mathcal{C}_{b p}$. This leads us to the fact that $c=\left(c_{r s}\right) \in\left(\Phi^{\star}\left(\mathcal{C}_{b p}\right)\right)^{\gamma}$ iff $O \in\left(\mathcal{C}_{b p}: \mathcal{M}_{u}\right)$. Hence, we achieve that $\left(\Phi^{\star}\left(\mathcal{C}_{b p}\right)\right)^{\gamma}=w_{1}$. The other part of the theorem can be proven by using similar technique. So, we omit it. 
Theorem 3.3. Consider the sets $w_{2}-w_{13}$ defined by

$$
\begin{aligned}
& w_{2}=\left\{c=\left(c_{r s}\right) \in \Omega: \exists b_{t u} \in \mathbb{C} \ni \vartheta-\lim _{r, s \rightarrow \infty} \sigma(r, s, t, u, m, n)=b_{t u}\right\}, \\
& w_{3}=\left\{c=\left(c_{r s}\right) \in \Omega: \exists L \in \mathbb{C} \ni \vartheta-\lim _{r, s \rightarrow \infty} \sum_{t, u} \sigma(r, s, t, u, m, n)=L \quad \text { exists }\right\} \text {, } \\
& w_{4}=\left\{c=\left(c_{r s}\right) \in \Omega: \exists u_{0} \in \mathbb{N} \ni \vartheta-\lim _{r, s \rightarrow \infty} \sum_{t}\left|\sigma\left(r, s, t, u_{0}, m, n\right)-b_{t u_{0}}\right|=0\right\} \text {, } \\
& w_{5}=\left\{c=\left(c_{r s}\right) \in \Omega: \exists t_{0} \in \mathbb{N} \ni \vartheta-\lim _{r, s \rightarrow \infty} \sum_{u}\left|\sigma\left(r, s, t_{0}, u, m, n\right)-b_{t_{0} u}\right|=0\right\}, \\
& w_{6}=\left\{c=\left(c_{r s}\right) \in \Omega: \forall t \in \mathbb{N}, \exists u_{0} \in \mathbb{N} \ni \sigma(r, s, t, u, m, n)=0, \forall u>u_{0}, \forall r, s \in \mathbb{N}\right\} \text {, } \\
& w_{7}=\left\{c=\left(c_{r s}\right) \in \Omega: \forall u \in \mathbb{N}, \exists t_{0} \in \mathbb{N} \ni \sigma(r, s, t, u, m, n)=0, \forall t>t_{0}, \forall r, s \in \mathbb{N}\right\} \text {, } \\
& w_{8}=\left\{c=\left(c_{r s}\right) \in \Omega: \exists u_{0} \in \mathbb{N} \ni \vartheta-\lim _{r, s \rightarrow \infty} \sum_{t} \sigma\left(r, s, t, u_{0}, m, n\right)=b_{u_{0}}\right\}, \\
& w_{9}=\left\{c=\left(c_{r s}\right) \in \Omega: \exists t_{0} \in \mathbb{N} \ni \vartheta-\lim _{r, s \rightarrow \infty} \sum_{u} \sigma\left(r, s, t_{0}, u, m, n\right)=b_{t_{0}}\right\} \text {, } \\
& w_{10}=\left\{c=\left(c_{r s}\right) \in \Omega: \exists b_{t u} \in \mathbb{C} \ni b p-\lim _{r, s \rightarrow \infty} \sum_{t, u}\left|\sigma(r, s, t, u, m, n)-b_{t u}\right|=0\right\}, \\
& w_{11}=\left\{c=\left(c_{r s}\right) \in \Omega: \forall t \in \mathbb{N} \ni b p-\lim _{r, s \rightarrow \infty} \sum_{u=1}^{s} \sigma(r, s, t, u, m, n) \quad \text { exists }\right\}, \\
& w_{12}=\left\{c=\left(c_{r s}\right) \in \Omega: \forall u \in \mathbb{N} \ni b p-\lim _{r, s \rightarrow \infty} \sum_{t=1}^{r} \sigma(r, s, t, u, m, n) \text { exists }\right\} \text {, } \\
& w_{13}=\left\{c=\left(c_{r s}\right) \in \Omega: \sum_{t, u}|\sigma(r, s, t, u, m, n)| \quad \text { converges }\right\} \text {. }
\end{aligned}
$$

In that case, following statements are satisfied:

(i) $\left(\Phi^{\star}\left(\mathcal{C}_{b p}\right)\right)^{\beta(\vartheta)}=\bigcap_{k=1}^{5} w_{k}$ ，

(ii) $\left(\Phi^{\star}\left(\mathcal{C}_{p}\right)\right)^{\beta(\vartheta)}=\bigcap_{k=1}^{3} w_{k} \cap w_{6} \cap w_{7}$ ，

(iii) $\left(\Phi^{\star}\left(\mathcal{C}_{r}\right)\right)^{\beta(\vartheta)}=\bigcap_{k=1}^{3} w_{k} \cap w_{8} \cap w_{9}$ ，

(iV) $\left(\Phi^{\star}\left(\mathcal{M}_{u}\right)\right)^{\beta(b p)}=w_{1} \cap w_{2} \bigcap_{k=10}^{13} w_{k}$ ，

(V) $\left(\Phi^{\star}\left(\mathcal{M}_{u}\right)\right)^{\beta(p)}=w_{2} \cap w_{6} \cap w_{7}$.

Proof.

(i) Suppose that $c=\left(c_{r s}\right) \in \Omega$ and $x=\left(x_{r s}\right) \in \Phi^{\star}\left(\mathcal{C}_{b p}\right)$. In that case, there exists a double sequence $y=\left(y_{r s}\right) \in \mathcal{C}_{b p}$ with $\Phi^{\star} x=y$. Since (3.16) holds, we deduce that $c x \in \mathcal{C} \mathcal{S}_{\vartheta}$ whenever $x \in \Phi^{\star}\left(\mathcal{C}_{b p}\right)$ iff $z \in \mathcal{C}_{\vartheta}$ whenever $y \in \mathcal{C}_{b p}$. This leads us to the fact that $c=\left(c_{r s}\right) \in\left(\Phi^{\star}\left(\mathcal{C}_{b p}\right)\right)^{\beta(\vartheta)}$ iff $O \in\left(\mathcal{C}_{b p}: \mathcal{C}_{\vartheta}\right)$. Therefore, the conditions of Lemma 3.1 are satisfied with $O=\left(o_{r s t u}\right)$ defined as in Theorem 3.2. Hence, we achieve that the $\beta(\vartheta)$-dual of the space $\Phi^{\star}\left(\mathcal{C}_{b p}\right)$ is $\bigcap_{k=1}^{5} w_{k}$.

The other parts of the Theorem can be done analogously by using the Lemmas 3.2, 3.3, 3.5 and 3.7, respectively. So, we pass the details. 


\section{Charactarization of Some Classes of 4-Dimensional Matrices}

In the current section, we deal with some 4-dimensional matrix mapping classes related to the double sequence spaces $\Phi^{\star}\left(\mathcal{M}_{u}\right), \Phi^{\star}\left(\mathcal{C}_{p}\right), \Phi^{\star}\left(\mathcal{C}_{b p}\right)$ and $\Phi^{\star}\left(\mathcal{C}_{r}\right)$ by using dual summability methods for double sequences which have been presented and examined by Başar [4] and Yeşilkayagil and Başar [37] and which have been applied by Tug [36].

Theorem 4.1. Assume that the elements of 4-dimensional infinite matrices $B=\left(b_{r s t u}\right)$ and $H=\left(h_{r s t u}\right)$ are connected with the relation

$$
h_{r s t u}=\sum_{m=t, t \mid m}^{\infty} \sum_{n=u, u \mid n}^{\infty} \frac{\mu\left(\frac{m}{t}\right) \mu\left(\frac{n}{u}\right)}{\varphi(m) \varphi(n)} t u b_{r s m n} .
$$

Then, $B \in\left(\Phi^{\star}(\Psi): \Lambda\right)$ iff $B_{r s} \in\left[\Phi^{\star}(\Psi)\right]^{\beta(\vartheta)}$ for every $r, s \in \mathbb{N}$ and $H \in(\Psi: \Lambda)$, where $\Psi$ and $\Lambda \in\left\{\mathcal{M}_{u}, \mathcal{C}_{p}, \mathcal{C}_{b p}, \mathcal{C}_{r}\right\}$.

Proof. Assume that $B \in\left(\Phi^{\star}(\Psi): \Lambda\right)$. In that case, $B x$ exists and is in $\Lambda$ for every $x \in \Phi^{\star}(\Psi)$ and it also implies that $B_{r s} \in\left[\Phi^{\star}(\Psi)\right]^{\beta(\vartheta)}$ for every $r, s \in \mathbb{N}$. Thus, we have the following equality derived from partial sums of the series $\sum_{t, u} b_{r s t u} x_{t u}$ with relation (2.4)

$$
\begin{aligned}
\sum_{t, u=1}^{i, j} b_{r s t u} x_{t u} & =\sum_{t, u=1}^{i, j} b_{r s t u}\left[\sum_{m|t, n| u} \frac{\mu\left(\frac{t}{m}\right) \mu\left(\frac{u}{n}\right)}{\varphi(t) \varphi(u)} m n y_{m n}\right] \\
& =\sum_{t, u=1}^{i, j}\left[\sum_{m=t, t \mid m}^{i} \sum_{n=u, u \mid n}^{j} \frac{\mu\left(\frac{m}{t}\right) \mu\left(\frac{n}{u}\right)}{\varphi(m) \varphi(n)} t u b_{r s m n}\right] y_{t u}
\end{aligned}
$$

for every $i, j \in \mathbb{N}$. In that case, if we take $\vartheta$-limit on equality above as $i, j \rightarrow \infty$, we have $B x=H y$. Therefore, we obtain that $H y \in \Lambda$ whenever $y \in \Psi$, that is $H \in(\Psi: \Lambda)$.

Conversely, suppose that $B_{r s} \in\left[\Phi^{\star}(\Psi)\right]^{\beta(\vartheta)}$ for every $r, s \in \mathbb{N}, H \in(\Psi: \Lambda)$ and $x \in \Phi^{\star}(\Psi)$ such that $y=\Phi^{\star} x$. In that case, $B x$ exists and therefore, the $(k, l)$ th rectangular partial sums of the series $\sum_{t, u} b_{r s t u} x_{t u}$ obtained as

$$
\begin{aligned}
(B x)_{r s}^{[k, l]} & =\sum_{t, u=1}^{k, l} b_{r s t u} x_{t u} \\
& =\sum_{t, u=1}^{k, l} b_{r s t u}\left[\sum_{m|t, n| u} \frac{\mu\left(\frac{t}{m}\right) \mu\left(\frac{u}{n}\right)}{\varphi(t) \varphi(u)} m n y_{m n}\right] \\
& =\sum_{t, u=1}^{k, l}\left[\sum_{m=t, t \mid m}^{k} \sum_{n=u, u \mid n}^{l} \frac{\mu\left(\frac{m}{t}\right) \mu\left(\frac{n}{u}\right)}{\varphi(m) \varphi(n)} t u b_{r s m n}\right] y_{t u}
\end{aligned}
$$

for every $r, s, k, l \in \mathbb{N}$. By taking $\vartheta$-limit on (4.2) while $k, l \rightarrow \infty$, it can be easily obtain from the following equality

$$
\sum_{t, u} b_{r s t u} x_{t u}=\sum_{t, u} h_{r s t u} y_{t u}
$$

for every $r, s \in \mathbb{N}$ that $B x=H y$ which leads us to the fact that $B \in\left(\Phi^{\star}(\Psi): \Lambda\right)$.

Corollary 4.1. Suppose that $B=\left(b_{r s t u}\right)$ is a 4-dimensional matrix. In that case the following statements are satisfied:

(i) $B \in\left(\Phi^{\star}\left(\mathcal{C}_{p}\right): \mathcal{C}_{\vartheta}\right)$ iff the conditions (3.1)-(3.3), (3.6) and (3.7) are satisfied with $h_{r s t u}$ in place of $b_{r s t u}$

(ii) $B \in\left(\Phi^{\star}\left(\mathcal{C}_{b p}\right): \mathcal{C}_{\vartheta}\right)$ iff the conditions (3.1)-(3.5) are satisfied with $h_{r s t u}$ in place of $b_{\text {rstu }}$,

(iii) $B \in\left(\Phi^{\star}\left(\mathcal{C}_{b p}\right): \mathcal{M}_{u}\right)$ iff the condition (3.1) is satisfied with $h_{r s t u}$ in place of $b_{r s t u}$,

(iv) $B \in\left(\Phi^{\star}\left(\mathcal{C}_{r}\right): \mathcal{C}_{\vartheta}\right)$ iff the conditions (3.1)-(3.3), (3.8) and (3.9) are satisfied with $h_{r s t u}$ in place of $b_{r s t u}$

(v) $B \in\left(\Phi^{\star}\left(\mathcal{M}_{u}\right): \mathcal{C}_{b p}\right)$ iff the conditions (3.1), (3.2), (3.10)-(3.13) are satisfied with $h_{r s t u}$ in place of $b_{r s t u}$ 
(vi) $B \in\left(\Phi^{\star}\left(\mathcal{M}_{u}\right): \mathcal{C}_{p}\right)$ iff the conditions (3.2), (3.6) and (3.7) are satisfied with $h_{r s t u}$ in place of $b_{r s t u}$.

Lemma 4.1. [40] Let $\Psi$ and $\Lambda$ be two double sequence spaces, $B=\left(b_{r s t u}\right)$ be any 4-dimensional matrix and $F=\left(f_{r s t u}\right)$ also be a 4-dimensional triangle matrix such that $f_{r s t u}=0$ if $t>r$ and $u>s$ for every $r, s, t, u \in \mathbb{N}$. In that case, $B \in\left(\Psi: \Lambda_{F}\right)$ iff $F B \in(\Psi: \Lambda)$.

Now, let us define the 4-dimensional matrix $G=\left(g_{r s t u}\right)$ by

$$
g_{r s t u}=\sum_{m|r, n| s} \phi_{r s m n}^{\star} b_{m n t u}
$$

for every $r, s, t, u \in \mathbb{N}$ and give following corollary.

Corollary 4.2. Suppose that $B=\left(b_{r s t u}\right)$ is a 4-dimensional matrix. In that case the following statements are satisfied:

(i) $B \in\left(\mathcal{C}_{p}: \Phi^{\star}\left(\mathcal{C}_{\vartheta}\right)\right)$ iff the conditions (3.1)-(3.3), (3.6) and (3.7) are satisfied with $g_{\text {rstu }}$ in place of $b_{\text {rstu }}$

(ii) $B \in\left(\mathcal{C}_{b p}: \Phi^{\star}\left(\mathcal{C}_{\vartheta}\right)\right)$ iff the conditions (3.1)-(3.5) are satisfied with $g_{r s t u}$ in place of $b_{\text {rstu }}$,

(iii) $B \in\left(\mathcal{C}_{r}: \Phi^{\star}\left(\mathcal{C}_{\vartheta}\right)\right)$ iff the conditions (3.1)-(3.3), (3.8) and (3.9) are satisfied with $g_{r s t u}$ in place of $b_{r s t u}$,

(iv) $B \in\left(\mathcal{L}_{p}: \Phi^{\star}\left(\mathcal{C}_{b p}\right)\right)$ iff the conditions (3.2) and (3.14) are satisfied for $0<p \leq 1$ and $\vartheta=b p$ with $g_{r s t u}$ in place of $b_{r s t u}$,

(v) $B \in\left(\mathcal{L}_{p}: \Phi^{\star}\left(\mathcal{C}_{b p}\right)\right)$ iff the conditions (3.2) and (3.15) are satisfied for $1<p<\infty$ and $\vartheta=b p$ with $g_{r s t u}$ in place of $b_{r s t u}$,

(vi) $B \in\left(\mathcal{L}_{p}: \Phi^{\star}\left(\mathcal{M}_{u}\right)\right)$ iff the condition (3.14) is satisfied for $0<p \leq 1$ with $g_{r s t u}$ in place of $b_{r s t u}$,

(vii) $B \in\left(\mathcal{L}_{p}: \Phi^{\star}\left(\mathcal{M}_{u}\right)\right)$ iff the condition (3.15) is satisfied for $1<p<\infty$ with $g_{r s t u}$ in place of $b_{r s t u}$

(viii) $B \in\left(\mathcal{M}_{u}: \Phi^{\star}\left(\mathcal{C}_{b p}\right)\right)$ iff the conditions (3.1),(3.2),(3.10)-(3.13) are satisfied with $g_{r s t u}$ in place of $b_{r s t u}$

(ix) $B \in\left(\mathcal{M}_{u}: \Phi^{\star}\left(\mathcal{C}_{p}\right)\right)$ iff the conditions (3.2), (3.6) and (3.7) are satisfied with $g_{\text {rstu }}$ in place of $b_{\text {rstu }}$

(x) $B \in\left(\mathcal{C}_{b p}: \Phi^{\star}\left(\mathcal{M}_{u}\right)\right)$ iff the condition (3.1) is satisfied with $g_{r s t u}$ in place of $b_{r s t u}$.

\section{References}

[1] Adams, C.R.: On non-factorable transformations of double sequences. Proc. Natl. Acad. Sci. USA. 19 (5), 564-567 (1933).

[2] Alp, P.Z., İlkhan, M.: On the difference sequence space $\ell_{p}\left(\hat{T}^{p}\right)$. Math. Sci. Appl. E-Notes. 7 (2), 161-173 (2019).

[3] Altay, B., Başar, F.: Some new spaces of double sequences. J. Math. Anal. Appl. 309 (1), 70-90 (2005).

[4] Bașar, F.: Summability Theory and Its Applications. Bentham Science Publishers. e-book. Monographs. Istanbul (2012).

[5] Başar, F., Sever, Y.: The space $\mathcal{L}_{q}$ of double sequences. Math. J. Okayama Univ. 51, 149-157 (2009).

[6] Boss, J.: Classical and Modern Methods in Summability. Oxford University Press. Newyork, (2000).

[7] Candan, M.: A New Approarch on the Spaces of Generalized Fibonacci Difference Null and Convergent Sequences. Math. Aeterna. 5 (1), 191-210 (2015).

[8] Candan, M., Güneş, A.: Paranormed sequence space of non-absolute type founded using generalized difference matrix. Proceedings of the National Academy of Sciences. India Section A. 85 (2), 269-276 (2015).

[9] Candan, M.: Some new sequence spaces derived from the spaces of bounded, convergent and null sequences. Int. J. Mod. Math. Sci. 12 (2), 74-87 (2014).

[10] Candan, M.: A new perspective on paranormed Riesz sequence space of non-absolute type. Global J. Math. Anal. 3 (4), 150-163 (2015).

[11] Cooke, R.C.: Infinite Matrices and Sequence Spaces. Macmillan and Co. Limited. London (1950).

[12] Çakan, C., Altay, B., Mursaleen, M.: The $\sigma$-convergence and $\sigma$-core of double sequences. Appl. Math. Lett. 19, 387-399 (2006). 
[13] Demiriz, S., Duyar, O.: Domain of the Cesàro mean matrix in some paranormed spaces of double sequences. Contemp. Anal. Appl. Math. 3 (2), 247-262 (2015).

[14] Demiriz, S., Erdem, S.: Domain of Euler-Totient Matrix Operator in the Space $\mathcal{L}_{p}$. Korean J. Math. 28 (2), 361-378 (2020).

[15] Güleç, G.C.H., İlkhan, M.: A New Paranormed Series Space Using Euler Totient Means and Some Matrix Transformations. Korean J. Math. 28 (2), 205-221 (2020).

[16] Hamilton, H.J.: Transformations of multiple sequences. Duke Math. J. 2, 29-60 (1936).

[17] Illkhan, M.: Matrix domain of a regular matrix derived by Euler totient function in the spaces $c_{0}$ and $c$. Mediterr. J. Math. 17, Article No: 27 (2020).

[18] İlkhan, M., Kara, E. E.: A New Banach Space Defined by Euler Totient Matrix Operator. Operators and Matrices. 13 (2), 527-544 (2019).

[19] İlkhan, M., Demiriz, S., Kara, E. E.: A new paranormed sequence space defined by Euler totient matrix. Karaelmas Science and Engineering Journal. 9 (2), 277-282 (2019).

[20] Kara, E. E.: Some topological and geometrical properties of new Banach sequence spaces. J. Inequal. Appl. 38, 15 pages (2013).

[21] Kara, E.E., İlkhan, M.: On some Banach sequence spaces derived by a new band matrix. British Journal of Mathematics and Computer Science. 9 (2), 141-159 (2015).

[22] Kara, E.E., İlkhan, M.: Some properties of generalized Fibonacci sequence spaces. Linear and Multilinear Algebra. 64 (11), 2208-2223 (2016).

[23] Kılınç, G., Candan, M.: Some generalized Fibonacci difference spaces defined by a sequence of modulus functions. Facta Universitatis. Series: Mathematics and Informatics. 32 (1), 95-116 (2017).

[24] Kovac, E.: On $\varphi$ convergence and $\varphi$ density. Mathematica Slovaca. 55, 329-351 (2005).

[25] Mòricz, F.: Extensions of the spaces $c$ and c from single to double sequences. Acta Math. Hungar. 57, 129-136 (1991).

[26] Mursaleen, M.: Almost strongly regular matrices and a core theorem for double sequences. J. Math. Anal. Appl. 293 (2), 523-531 (2004).

[27] Mursaleen, M., Bașar, F.: Domain of Cesàro mean of order one in some spaces of double sequences. Stud. Sci. Math. Hungar. 51 (3), 335-356 (2014).

[28] Mursaleen, M., Mohiuddine, S. A.: Convergence Methods for Double Sequences and Applications. Springer. New Delhi. Heidelberg. New York. Dordrecht. London (2014).

[29] Niven, I., Zuckerman, H.S., Montgomery, H.L.: An introduction to the theory of numbers. (5. Edition). Wiley, New York (1991).

[30] Pringsheim, A.: Zur Theorie der zweifach unendlichen Zahlenfolgen. Math. Ann. 53, 289-321 (1900).

[31] Robison, G. M.: Divergent double sequences and series. Amer. Math. Soc. Trans. 28, 50-73 (1926).

[32] Schaefer, H.H.: Topological Vector Spaces. Graduate Texts in Matematics. 3. 5th printing (1986).

[33] Schoenberg, I.: The integrability of certain functions and related summability methods. The American Monthly. 66, 361-375 (1959).

[34] Stieglitz, M., Tietz, H.: Matrix transformationen von folgenraumen eine ergebnisbersicht. Mathematische Zeitschrift. 154, 1-16 (1977).

[35] Talebi, G.: Operator norms of four-dimensional Hausdorff matrices on the double Euler sequence spaces. Linear and Multilinear Algebra. 65 (11), 2257-2267 (2017). 
[36] Tug̃g, O.: Four-dimensional generalized difference matrix and some double sequence spaces. J. Inequal. Appl. 2017, 149 (2017).https://doi.org/10.1186/s13660-017-1423-y

[37] Yeşilkayagil, M., Başar, F.: Four dimensional dual and dual of some new sort summability methods. Contemp. Anal. Appl. Math. 3 (1), 13-29 (2015).

[38] Yeşilkayagil, M., Başar, F.: Mercerian theorem for four dimensional matrices. Commun. Fac. Sci. Univ. Ank. Ser. A1. 65 (1), 147-155 (2016).

[39] Yeşilkayagil, M., Başar, F.: On the characterization of a class of four dimensional matrices and Steinhaus type theorems. Kragujev. J. Math. 40 (1), 35-45 (2016).

[40] Yeşilkayagil, M., Başar, F.: Domain of Riesz Mean in the Space $\mathcal{L}_{s}$. Filomat. 31 (4), 925-940 (2017).

[41] Zeltser, M.: Investigation of double sequence spaces by soft and hard analitic methods. Dissertationes Mathematicae Universtaties Tartuensis. Tartu University Press. Univ. of Tartu. Faculty of Mathematics and Computer Science. 25, Tartu, (2001).

[42] Zeltser, M.: On conservative matrix methods for double sequence spaces. Acta Math. Hung. 95 (3), 225-242 (2002).

[43] Zeltser, M., Mursaleen, M., Mohiuddine, S. A.: On almost conservative matrix mathods for double sequence spaces. Publ. Math. Debrecen. 75, 387-399 (2009).

\section{Affiliations}

\section{SEZER ERDEM}

ADDRESS: Farabi Anatolian Imam Hatip High School, 44400, Malatya-Turkey. E-MAIL: sezererdem8344@gmail.com

ORCID ID:0000-0001-9420-8264

\section{SERKAN DEMIRIZ}

ADDRESS: Tokat Gaziosmanpaşa University, Department of Mathematics, 60250, Tokat-Turkey. E-MAIL: serkandemiriz@gmail.com

ORCID ID:0000-0002-4662-6020 